\title{
Ultra-High-Throughput Multi-Parametric Imaging Flow Cytometry
}

\author{
Andrew deMello*, Anand Rane, Gregor Holzner, and Stavros Stavrakis \\ Department of Chemistry \& Applied Biosciences, ETH Zürich, Vladimir Prelog Weg 1, 8093 Zürich, Switzerland
}

\begin{abstract}
I will present a microfluidic imaging flow cytometer incorporating stroboscopic illumination, for blur-free cellular analysis at throughputs exceeding 100,000 cells per second. By combining passive (inertial or viscoelastic) focusing of cells in parallel microchannels with stroboscopic illumination, such chip-based cytometers are able to extract multi-colour fluorescence and bright-field images of single cells moving at high linear velocities. This in turn allows accurate sizing of individual cells, intracellular localization and analysis of heterogeneous cell suspensions. The method is showcased through the rapid enumeration of apoptotic cells, high-throughput discrimination cell cycle phases and localization of p-bodies.
\end{abstract}

\section{Introduction}

Flow cytometry, based on point-based detection schemes, is recognized to be the gold-standard tool for highthroughput manipulation, processing and analysis of cells in flow, but is typically limited in regard to the number of cells that can be interrogated per unit time and the information yield per experiment. To address such limitations, much recent activity has focused on the development of imaging flow cytometry platforms[1]. Imaging flow cytometry combines the high-throughput capabilities of conventional flow cytometry with singlecell imaging and enables high information content analysis of large cellular populations. We have developed approaches for sheathless imaging flow cytometry that incorporate stroboscopic illumination for blur-free cellular analysis at throughputs exceeding 100,000 cells per second[2]. By combining inertial (or viscoelastic) focusing of cells in parallel (or single) microchannels and stroboscopic illumination, such chip-based cytometers are able to extract multi-colour fluorescence, bright-field, and dark-field images. This enables the accurate sizing of individual cells and the analysis of heterogeneous cell suspensions while maintaining operational simplicity..

\section{Method}

A schematic of one of microfluidic imaging flow cytometers is shown in Figure 1. In simple terms, the cytometer consists of three sub-components: an input module (R1) incorporating a series of posts to remove cellular aggregates prior to analysis, a cell-focusing manifold (R2) containing six parallel and winding channels (each with a width and depth of 40 and $22 \mathrm{~mm}$, respectively), and an imaging region (R3) where dualcolour fluorescence, bright-field, and dark-field images of the cell flows can be extracted. In addition to lateral focusing, fluid inertia is used to control the axial position of cells within each microfluidic channel and the intercellular spacings. To eliminate motion blur associated with CMOS cameras when imaging rapidly moving cells, we implement a stroboscopic illumination scheme and dual-view detection system, to extract blur-free images. Details of this approach have been reported elsewhere[3].

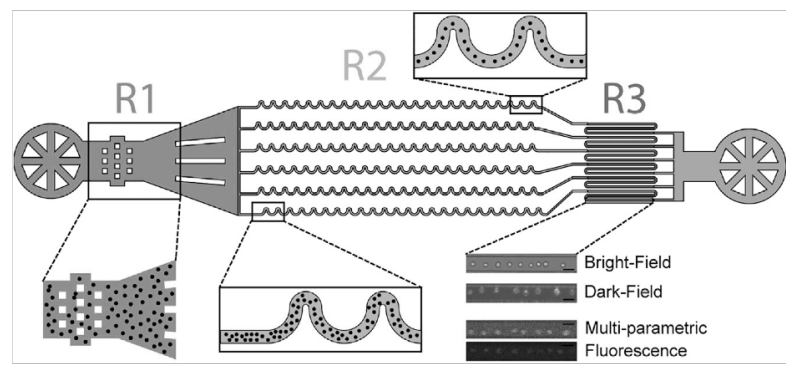

Fig. 1. Schematic of an inertial microfluidic flow cytometer highlighting the inlet port (blue), inertial focusing channels (orange), imaging area for the detection (green), and the outlet port for the collection (gray). (R1) Filter for removing the cell debris, cell aggregates, and the clumps. (R2) Schematic of the cells flowing at the beginning and at the end of the inertial focusing channel. (R3) Representative images from all the possible imaging modalities of the detection. All scale bars represent $30 \mathrm{~mm}$. Image adapted and reproduced from [2].

\section{Results}

My talk will describe how we have applied the method to the rapid enumeration of apoptotic cells, high-throughput discrimination of cell cycle phases and the localization of p-bodies inside cells[2]. Our approach makes significant contributions to the large-scale screening of cells, allows quantitative analyses of heterogeneous cell populations, and enables the systematic mapping of molecular interactions within real-world cell populations.

\section{References}

1. S. Stavrakis, G. Holzner, J. Choo \& A.J. deMello, Current Opinion in Biotechnology 55, 36 (2018)

2. A.S. Rane, J. Rutkauskaite, A.J. deMello \& S. Stavrakis, Chem 3, 588 (2017)

3. D. Hess, A.S. Rane, A.J. deMello \& S. Stavrakis, Analytical Chemistry 87, 4965 (2015)

\footnotetext{
* Corresponding author: andrew@ethz.ch
} 